\title{
SUBMICROSCOPIC CHROMOSOMAL VARIATIONS IN CHILDREN WITH IDIOPATHIC INTELLECTUAL AND DEVELOPMENTAL DISABILITIES
}

\author{
NEETHA JOHN ${ }^{1}$, SHARMA PSVN ${ }^{2}$, RAJASEKHAR MOKA ${ }^{1 *}$ \\ ${ }^{1}$ Department of Cell and Molecular Biology, Manipal School of Life Sciences, Manipal Academy of Higher Education, Manipal, Karnataka, \\ India. ${ }^{2}$ Department of Psychiatry, Kasturba Medical College, Manipal Academy of Higher Education, Manipal, Karnataka, India.
}

Email: rsmoka@gmail.com

Received: 19 November 2019, Revised and Accepted: 24 December 2019

ABSTRACT

Objective: Intellectual disability is the most common developmental disorder that originates before the age of 18 years and is characterized by limitation in intellectual functioning and adaptive behaviour. The fact that $>30$ to $50 \%$ of all causes are still unknown in etiology is increasing the burden of the clinical evaluators and managers handling these children. The purpose of this study was to have an optimal genetic diagnostic evaluation to assist paediatricians in providing medical advice for children with intellectual disabilities and global developmental delays.

Methods: The study was initiated with 385 cases; however, only 201 cases had no cytogenetic abnormality and negative for PCR test for FXS. However, these subjects showed characteristic signs of facial dysmorphisms, developmental delay, mild to severe intellectual disability, which were unique and unspecific with lack of major hallmarks for any particular syndrome/phenotype, considered as "idiopathic" and tested for MLPA analysis and subsequently confirmed by FISH and RT-qPCR.

Results: A total of $23(11.44 \%)$ cases were found to have submicroscopic chromosomal variations [microdeletions (18 cases), microduplications (5 cases)]. We categorized the aberrations detected in these cases as novel and as variants of uncertain significance. All these cases showed clear evidence for segregation of the variation and were provided with the required genetic counselling.

Conclusion: MLPA method gives a better yield in combination with karyotype analysis. The detection rate as per current analysis suggests that the use of MLPA could be a robust, high throughput yet cost-effective technique for use in a diagnostic set up.

Keywords: Intellectual disabilities, Global developmental delay, Chromosomal variations, Copy number variations.

(C) 2020 The Authors. Published by Innovare Academic Sciences Pvt Ltd. This is an open access article under the CC BY license (http://creativecommons. org/licenses/by/4. 0/) DOI: http://dx.doi.org/10.22159/ajpcr.2020.v13i2.36405

\section{INTRODUCTION}

Intellectual and developmental disabilities (IDDs) affects 1-3\% of the general population and represents an important health burden in developing countries. Clinical diagnosis of IDD is based on intelligent quotient (IQ <70) and substantially limitation in one or more daily activities. Global developmental delay (GDD; children younger, i.e., <5 years) and intellectual disability (ID; children $>5$ years of age) are common concerns and are caused by multifactor that impairs the developmental milestones such as growth and cognitive development [1]. Genetic causes include chromosomal, monogenic/polygenic variations, and copy number changes [2]. The majority of the cases (25-50\%) reported having genetic etiologies such as chromosomal syndromes, microdeletions, and gene mutations and more than $50 \%$ of its still unexplained $[3,4]$. Considering their severity in IQ and congenital anomaly, IDDs are grouped into syndromic and non-syndromic forms. Syndromic IDD presents with one or more clinical phenotypes or comorbidities in addition to ID. However, nonsyndromic IDD has a clear definition with no comorbidities [5]. In the present study, conventional cytogenetics was used for initial screening of 385 clinically diagnosed children ( $<18$ years) with unknown etiology. Among them, 201 children were shown to have no gross chromosomal abnormalities. These 201 children were deemed as idiopathic intellectual developmental disabilities (idiopathic IDDs) and further assessed submicroscopic variations or copy number changes using multiplex ligation-dependent probe amplification (MLPA) assay [6]. We henceforth, intended that MLPA is high throughput and cost-effective and has a reduced turnaround time to diagnose syndromic and nonsyndromic IDDs.

\section{METHODS}

Clinically diagnosed children with ID and GDD with no known etiology were recruited to investigate submicroscopic and subtelomeric chromosomal variations. Three hundred and eighty-five subjects were recruited after the Institutional Ethics Committee approval and informed consent from parents or guardians, and clinical profiles were noted. Peripheral blood (5 ml) was collected in a green top Vacutainer from each patient to carry out cytogenetics as well as molecular analysis. Lymphocytes were cultured and the metaphase plates prepared as per the standard operating procedure [7]. The metaphase slides were trypsinized and stained with Giemsa stain, visualized and scored using a Zeiss microscope and IKAROS software. A minimum of 50 metaphases was analyzed using Ikaros Karyotyping System.

Genomic DNA was isolated from a peripheral blood sample using a QIAGEN genomic DNA isolation kit according to the manufacturer's instruction. All samples were processed for MLPA probe sets (SALSA P036 and P070) according to the manufacturer's (MRC-Holland) instructions with minor modifications. The selection criteria for $\mathrm{X}$ chromosome screening included males with a normal MLPA profile using SALSA P245A2 and MRX106B1 probe sets. The amplified probes corresponding to multiple genomic locations have different lengths and were then size separated by capillary electrophoresis. Each peak represents a genomic locus and peak height represented the product produced which corresponds to the copy number. The data thus obtained are transported to a peak pattern analysis platform where the peaks of the heights are normalized, and the dosage quotient was calculated. The data further validated by quantitative real-time polymerase chain reaction (RT-qPCR) using SYBR green assay. 


\section{RESULTS}

A cohort of 385 clinical subjects was recruited as referred by clinicians from Kasturba Hospital and in and around primary health-care centers and schools for the specially abled based on unknown etiology of the clinical features with intellectual disability, developmental delay, with and without dysmorphic features (of the face including eyes, ears, and nose), congenital abnormalities (hands, brain, and abnormal genitalia), abnormality in postnatal growth (growth retardation or overgrowth), behavioral disorders (autistic spectrum of disorders, hyperactivity disorders, disturbed sleep patterns, aggressive behaviors, and self-injurious nature), or a reported significant family history of IDD. Further, for selective inclusion to the study, additional clinical selection criteria based on the checklist adapted from BB de Vries group published in 2001 [8]. In this study, 385 clinically diagnosed ID/developmental delays were included and subjected to the first line of screening by conventional karyotype after G-banding using trypsin and Giemsa stain. All cases that showed normal chromosomal coordinates were then subjected to test for Fragile X syndrome screening by PCR and only those subjects $(n=201)$ showed that negative results for these two screening tests were analyzed using MLPA. Among them, 23 cases were detected to have submicroscopic aberrations (19 deletions, five duplications). The results are summarized in Table 1.

These variations have been categorized into three classes, "Pathogenic," "Likely pathogenic," and "copy number variants (CNVs) of unknown significance." Pathogenic CNVs are those variations which have been reported previously in the literature and have been implicated in the disease with phenotypes consistent with the previous reports. We report novel and likely pathogenic CNVs in the vicinity of reported CNVs (same protein domain, gene locus) $[9,10]$ that can have major disease implications as found in the literature. CNVs of unknown significance are classified as those which have less functional implications but appear in association with the disease. Three microdeletions detected were thought to be pathogenic;

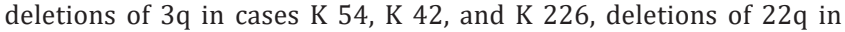
cases K 19, K 71, K 112, and deletions in Xq-in cases K 58 and K123. These deletions have already been reported in the literature to be pathogenic and have a very clear set of clinical features associated with 3q29 microdeletion syndrome, Phelan-McDermid syndrome (PMS) 22q13, and Xq28 deletion syndrome. However, the SLC6A8 deletion in case K58, SHANK3 deletion in K 71, and DLG1 deletion in case K42 and K226 could not be reproduced by qRT-PCR and hence have been eliminated from the final list of abnormalities.

A deletion of 22q11.21 in K 25 was detected, which was found to be inherited from a phenotypically healthy parent; hence, this variation was assumed to be benign. Further, a duplication of the MeCP2 gene was detected in a patient who also inherited the variation from a healthy parent and had two siblings who had normal intellectual and behavioral phenotypes. Thus, this variation could not be ascertained as disease causing. This could also be due to the lack of literary evidence to substantiate this finding. In remaining cases, parental samples were not available and hence an inheritance pattern could not be established. We classify the aberrations detected in these cases as novel and as variants of uncertain significance. All cases which showed clear evidence for segregation of the variation were provided with the required genetic counseling.

\section{DISCUSSION}

All diagnosed individuals/children $(n=201,52.2 \%)$ found to have normal chromosomal complement were further processed for copy number rearrangement screening by MLPA. At present, there is limited information from South Indian population about the diagnostic applicability of MLPA in the detection of cryptic chromosomal abnormalities causing IDDs. To the best of our knowledge, our study may be the first one to report the clinical use of MLPA in detecting IDDs in a clinical diagnostic setup with an initial set of 122 samples from the region of North Kerala and Karnataka [11]. Further, we extended the study into 201 isolated idiopathic subjects among 385 recruited IDDs. In 2014, the clinical utility of MLPA in 203 unexplained mental retardations was reported by Boggula et al. [12]. Subsequently, several groups have started in using MLPA in a large-scale diagnostic as well as in research.

SHANK3 on 22q13 deletion was reported in association with PMS, with characteristic features include developmental delay, ID, hypotonia, and delayed speech. Our results in concordance with literature, it was found that SHANK3 gene microdeletion is the common cause of autistic spectrum and mild ID [13]. We identified a microdeletion at a subtelomeric region of 22q13 in three children with developmental delay including facial dysmorphism and language deficit. The deletion of SHANK3 was the first most likely to cause the neurobehavioral symptoms of PMS and has been reported to substantiate gene dosage-

Table 1: A detailed clinical profile and copy number changes in patients with IDDs

\begin{tabular}{|c|c|c|c|c|c|c|c|c|c|c|}
\hline $\begin{array}{l}\text { Case no./ } \\
\text { Age/Sex }\end{array}$ & $\begin{array}{l}\text { Maternal } \\
\text { age }(Y)\end{array}$ & $\begin{array}{l}\text { Socioeconomic } \\
\text { status }\end{array}$ & Consanguinity & IQ & $\begin{array}{l}\text { Family } \\
\text { history }\end{array}$ & $\begin{array}{l}\text { Clinical } \\
\text { features }\end{array}$ & Type & Transmission & $\begin{array}{l}\text { Gene } \\
\text { implicated }\end{array}$ & $\begin{array}{l}\text { Chr. } \\
\text { location }\end{array}$ \\
\hline $\mathrm{K} 1 / 3 \mathrm{Y} / \mathrm{F}$ & 28 & III & No & - & Sporadic & GDD & Del & De novo & LIMK1 & $7 q 11.23$ \\
\hline K19/4Y/M & 30 & IV & No & - & Unknown & DD & Del & Unknown & SHANK3 & $22 \mathrm{q} 13.33$ \\
\hline $\mathrm{K} 25 / 2 \mathrm{Y} / \mathrm{M}$ & 24 & IV & Yes & - & Sporadic & Di-George & Del & Unknown & SNAP29 & $22 \mathrm{q} 11.21$ \\
\hline K42/1Y/M & 23 & V & Yes & - & Sporadic & DD/Dys & Del & De novo & $D L G 1$ & $3 q 29$ \\
\hline K54/17Y/M & 35 & IV & No & 55 & Unknown & MID/Dys & Del & Unknown & $D L G 1$ & $3 q 29$ \\
\hline K58/8M/M & 22 & V & Yes & - & Sporadic & Dys/DD & Del & De novo & SLC6A8 & Xq28 \\
\hline $\mathrm{K} 71 / 3 \mathrm{Y} / \mathrm{F}$ & 24 & III & No & - & Sporadic & ID & Del & De novo & SHANK3 & $22 \mathrm{q} 13.33$ \\
\hline $\mathrm{K} 92 / 5 \mathrm{Y} / \mathrm{F}$ & 32 & VI & Yes & 50 & Familial & ID/Dys & Del & Maternal & MECP2 & $\mathrm{Xq} 28$ \\
\hline K112/12Y/M & 28 & IV & No & 54 & Sporadic & ID & Del & De novo & SHANK3 & $22 \mathrm{q} 13.33$ \\
\hline $\mathrm{K} 153 / 1.5 \mathrm{Y} / \mathrm{F}$ & 24 & III & No & - & Unknown & Microcephaly & Dup & Unknown & MECP2 & $\mathrm{Xq} 28$ \\
\hline $\mathrm{K} 59 / 14 \mathrm{Y} / \mathrm{F}$ & 28 & III & No & 67 & Sporadic & ID/Dys & Del & De novo & CAPN10 & 2qter \\
\hline K77/8Y/F & 32 & VI & Yes & 53 & Sporadic & GDD/Dys & Del & De novo & SOX12 & 20pter \\
\hline K79/18Y/M & 23 & VI & Yes & 66 & Sporadic & MID & Del & De novo & MTA1 & 14qter \\
\hline K91/7Y/M & 36 & III & No & 60 & Sporadic & IID & Del & De novo & FBX025 & 8pter \\
\hline K100/9Y/M & 28 & III & No & 57 & Sporadic & ID/Dys & Del & De novo & CHL1 & 3pter \\
\hline K120/7Y/M & 25 & VI & Yes & 63 & Unknown & ID/ASD & Dup & Unknown & $R A B L 2 B$ & 22qter \\
\hline K226/3Y/F & 27 & III & No & - & Unknown & DD & Del & Unknown & $D L G 1$ & $3 q 29$ \\
\hline K235/9Y/M & - & - & No & - & Unknown & William's & Del & Unknown & $L I M K 1 / E L N$ & $7 q 11.23$ \\
\hline $\mathrm{K} 107 / 11 \mathrm{Y} / \mathrm{M}$ & 29 & III & No & 54 & Unknown & ID & Dup & De novo & $R B M 11$ & 21qter \\
\hline $\mathrm{K} 111 / 9 \mathrm{Y} / \mathrm{F}$ & 30 & IV & Yes & 45 & Sporadic & ID & Dup & De novo & GNB2L1 & 5qter \\
\hline $\mathrm{K} 123 / 4 \mathrm{Y} / \mathrm{F}$ & 29 & III & No & 55 & Unknown & ID & Del & De novo & SLC6A8 & Xq28 \\
\hline
\end{tabular}

Y: Years; M: Male; F: Female; Del: deletion; DD: Developmental delay, ID: Intellectual disability, MID: Mild ID, ASD: Autistic spectrum of diseases, Dys: Dysmorphology, GDD: Global developmental delay 
sensitive synaptic pathway and important regulator or postsynaptic membrane functionalities in ID and autism $[14,15]$

Williams syndrome is a multisystem neurodevelopmental disorder caused by the deletion of contiguous genes. In this MLPA analysis, we detected two Williams syndromes diagnosed patients with a microdeletion at 7q11.2 for ELN and LIMK1 genes. In a study on familial and sporadic IDs, deletion in both ELN and LIMK1 was detected in an 8-year-old intellectual disabled patient and considered to be a pathogenic marker for Williams-Beuren syndrome [16]. Early studies already implicated that these two genes are important in causing some characteristics of cognitive profiles since the LIMK1 gene is important for long-term memory and synaptic plasticity through transcriptional factor cellular retinol-binding protein in normal individual [17]. In this study, we have detected SEMA7A microdeletion in 14 years' female who had growth retardation with distinct facial features ID and with chromosome 15q24 microdeletion syndrome that is a rare and recently reported in 19 individuals [18]. The clinical phenotypes such as growth retardation, ID, and distinct facial features have been attributed to its role in brain development [19].

Variation in the DLG1 gene on 3q29 was detected in three of our cohort patients with mild-to-moderate ID with autistic phenotypes. Willatt et al. (2005) [20] were reported 3q29 microdeletion syndrome in six patients with ID and associated dysmorphic features, in which they specified the deletion encompasses 22 genes, including PAK2 and DLG1, which are autosomal homologs of two known X-linked MR genes, PAK3, and DLG3 [21]. Earlier Nakagawa et al. [22] on RNAi knockdown experiments have confirmed that the protein disc large homolog 1 (Drosophila) encoded by DLG1 is an important component of the postsynaptic density. In the present study, we recorded facial dysmorphic features with developmental delay in a male subject and MLPA analysis showed de novo deletion of 22q11.2 regions which harbor SNAP29 as one of the important genes involved in membrane trafficking. Bassett et al. [23] reported that 22q11.2 is the most common microdeletion syndrome associated with DiGeorge/velocardiofacial syndrome and with neurodevelopmental phenotype including ID. SNAP29 (synaptosome-associated protein, $29 \mathrm{kDa}$ ) is one of the key transcriptional proteins in neuronal plasticity in mice [24]. Variations in SNAP29 are accountable for unusual phenotypes in some patients with 22q11 deletions such as schizophrenia, autism, and ID [25,26]

MeCP2 on Xq28 deletions or duplications is usually associated with $\mathrm{X}$-linked neurodevelopmental disorder Rett syndrome which is one of the main causes of intellectual deficit in girls [27]. Female patients with Xq28 duplication are rare, whereas, in our cohort, we identified heterozygous de novo MeCP2 deletion and duplication in two different female subjects. However, both the subjects showed the same phenotypic features such as microcephaly, language delay, seizure and compulsive hand movements, and mild IQ. Loss- or gain-of-MeCP2 function has deleterious effects on dendritic morphology so that alterations in MECP2 expression have been shown to impact dendritic plasticity and this substantiates our findings [28]. We identified two subjects with similar Xq28 deletion with psychomotor retardation correlated with the involvement of SLC6A8 microdeletion, a creatinine transporter gene. Recent studies supported the association of cerebral creatine deficiency and intellectual disabilities $[29,30]$

Subtelomeric deletions in RBM11, GNB2L1, CAPN10, SOX12, MTA1, FBX025, and CHL1 and duplications in RABL2B and VAMP7 were also detected in our cohort patients, as indicated in Table 1. In 2004, Rooms et al. [31] reported 5.35 subtelomeric rearrangements in patients with mild-to-severe ID who had dysmorphic features and/or familial history. In another study on 132 ID subjects with associated dysmorphic features, nine chromosomal rearrangements corresponding to a diagnostic yield of $\sim 7 \%$ were detected. However, only five cases had imbalances which could be correlated to the phenotypes [32]. Telomere fluorescent in situ hybridization (FISH) revealed 11 subtelomeric abnormalities in ten patients, in a study, which also employed MLPA and array CGH for confirmation [33]. In a recent study, Wang et al. [34] reported 32 cases of subtelomeric rearrangements with a yield of a percentage of 21 in children with IDD in Chinese population.

\section{CONCLUSION}

In addition to conventional cytogenetics, molecular cytogenetic assays such as FISH and MLPA have gained popularity in developing countries for the proper diagnosis and management of idiopathic IDDs, which are also a substantial socioeconomic burden in these countries. For the past few decades, screening of gross chromosomal abnormalities pertaining to intellectual and associated anomalies has become a well-established testing strategy. However, a combination of karyotyping, MLPA, as well as next-generation sequencing might further help in finding both grosses and smaller variations in the genome.

\section{ACKNOWLEDGMENT}

This work was ostensibly supported by the Indian Council of Medical Research, New Delhi (F.No. 54/6/2008-HUM-BMS). We thank Director, School of Life Sciences, Manipal Academy of Higher Education, Manipal - 576 104, for his support and facilitated the infrastructure to complete this work. We are also thankful to clinicians and colleagues for their cooperation and assistance. Finally, our appreciation is due to the patients and their families.

\section{AUTHOR'S CONTRIBUTIONS}

All authors discussed the results and contributed to the final manuscript. Dr. Neetha John (SRF) - handling MLPA analysis and manuscript preparation. Dr. PSVN Sharma, psychiatrist, and specialist in autistic spectral disorders and intellectual disabilities - clinical diagnosis and review. Dr. Rajasekhar Moka - conceptualization in preparation of manuscript and review.

\section{CONFLICTS OF INTEREST}

The authors have no conflicts of interest to declare in relation to this article

\section{REFERENCES}

1. Curry CJ, Stevenson RE, Aughton D, Byrne J, Carey JC, Cassidy S, et al. Evaluation of mental retardation: Recommendations of a consensus conference: American College of Medical Genetics. Am J Med Genet 1997;72:468-77.

2. Karam SM, Riegel M, Segal SL, Félix TM, Barros AJ, Santos IS, et al. Genetic causes of intellectual disability in a birth cohort: A population-based study. Am J Med Genet A 2015;167:1204-14

3. Rauch A, Wieczorek D, Graf E, Wieland T, Endele S, Schwarzmayr T, et al. Range of genetic mutations associated with severe non-syndromic sporadic intellectual disability: An exome sequencing study. Lancet 2012;380:1674-82

4. Moeschler JB. Genetic evaluation of intellectual disabilities. Semin Pediatr Neurol 2008;15:2.

5. Kaufman L, Ayub M, Vincent JB. The genetic basis of non-syndromic intellectual disability: A review. J Neurodev Disord 2010;2:182-209.

6. Schouten JP, McElgunn CJ, Waaijer R, Zwijnenburg D, Diepvens F, Pals G. Relative quantification of 40 nucleic acid sequences by multiplex ligation-dependent probe amplification. Nucleic Acids Res 2002;30:e57.

7. Moorhead PS, Nowell PC, Mellman WJ, Battips DM, Hungerford DA. Chromosome preparations of leukocytes cultured from human peripheral blood. Exp Cell Res 1960;20:613-6.

8. de Vries BB, White SM, Knight SJ, Regan R, Homfray T, Young ID, et al. Clinical studies on submicroscopic subtelomeric rearrangements: A checklist. J Med Genet 2001;38:145-50.

9. Kearney HM, Thorland EC, Brown KK, Quintero-Rivera F, South ST. Working Group of the American College of Medical Genetics Laboratory Quality, Assurance Committee. American college of medical genetics standards and guidelines for interpretation and reporting of postnatal constitutional copy number variants. Genet Med 2011;13:680-5.

10. Leung IY, Pooh RK, Wang CC, Lau TK, Choy KW. Classification of pathogenic or benign status of CNVs detected by microarray analysis. 
Expert Rev Mol Diagn 2010;10:717-21.

11. John N, Rajasekhar M, Girisha KM, Sharma PS, Gopinath PM. Multiplex ligation-dependant probe amplification study of children with idiopathic mental retardation in South India. Indian J Hum Genet 2013;19:165-70

12. Boggula VR, Shukla A, Danda S, Hariharan SV, Nampoothiri S, Kumar R, et al. Clinical utility of multiplex ligation-dependent probe amplification technique in identification of aetiology of unexplained mental retardation: A study in 203 Indian patients. Indian J Med Res 2014;139:66-75.

13. Zwanenburg RJ, Ruiter SA, van den Heuvel ER, Flapper BC, Van Ravenswaaij-Arts CM. Developmental phenotype in Phelan-McDermid (22q13.3 deletion) syndrome: A systematic and prospective study in 34 children. J Neurodev Disord 2016;8:16.

14. Berkel S, Marshall CR, Weiss B, Howe J, Roeth R, Moog U, et al. Mutations in the SHANK2 synaptic scaffolding gene in autism spectrum disorder and mental retardation. Nat Genet 2010;42:489-91.

15. Chen J, Yu S, Fu Y, Li X. Synaptic proteins and receptors defects in autism spectrum disorders. Front Cell Neurosci 2014;8:276.

16. Rafati M, Seyyedaboutorabi E, Ghadirzadeh MR, Heshmati Y, Adibi $\mathrm{H}$ Keihanidoust Z, et al. "Familial" versus "Sporadic" intellectual disability: Contribution of common microdeletion and microduplication syndromes. Mol Cytogenet 2012;5:9.

17. Todorovski Z, Asrar S, Liu J, Saw NM, Joshi K, Cortez MA, et al. LIMK1 regulates long-term memory and synaptic plasticity via the transcriptional factor CREB. Mol Cell Biol 2015;35:1316-28.

18. Magoulas PL, El-Hattab AW. Chromosome 15q24 microdeletion syndrome. Orphanet J Rare Dis 2012;7:2

19. Mefford HC, Batshaw ML, Hoffman EP. Genomics, intellectual disability, and autism. N Engl J Med 2012;366:733-43.

20. Willatt L, Cox J, Barber J, Cabanas ED, Collins A, Donnai D, et al. 3q29 microdeletion syndrome: Clinical and molecular characterization of a new syndrome. Am J Hum Genet 2005;77:154-60.

21. Sagar A, Bishop JR, Tessman DC, Guter S, Martin CL, Cook EH. Co-occurrence of autism, childhood psychosis, and intellectual disability associated with a de novo 3q29 microdeletion. Am J Med Genet A 2013;161A:845-9.

22. Nakagawa T, Futai K, Lashuel HA, Lo I, Okamoto K, Walz T, et al. Quaternary structure, protein dynamics and synaptic function of SAP97 controlled by L27 domain interaction. Neuron 2004;44:453-67.

23. Bassett AS, McDonald-McGinn DM, Devriendt K, Digilio MC, Goldenberg P, Habel A, et al. Practical guidelines for managing patients with 22q11.2 deletion syndrome. J Pediatr 2011;159:332-90.
24. Pollak DD, Herkner K, Hoeger H, Lubec G. Behavioral testing upregulates pCaMKII, BDNF, PSD-95 and egr-1 in hippocampus of FVB/N mice. Behav Brain Res 2005;163:128-35.

25. McDonald-McGinn DM, Fahiminiya S, Revil T, Nowakowska BA, Suhl J, Bailey A, et al. Hemizygous mutations in SNAP29 unmask autosomal recessive conditions and contribute to atypical findings in patients with 22q11.2DS. J Med Genet 2013;50:80-90.

26. Friedman J, Adam S, Arbour L, Armstrong L, Baross A, Birch P, et al. Detection of pathogenic copy number variants in children with idiopathic intellectual disability using $500 \mathrm{~K}$ SNP array genomic hybridization. BMC Genomics 2009;10:526.

27. Scala E, Longo I, Ottimo F, Speciale C, Sampieri K, Katzaki E, et al. MECP2 deletions and genotype-phenotype correlation in Rett syndrome. Am J Med Genet A 2007;143A:2775-84

28. Na ES, Nelson ED, Kavalali ET, Monteggia LM. The impact of MeCP2 loss- or gain-of-function on synaptic plasticity. Neuropsychopharmacology 2013;38:212-9.

29. Stockler-Ipsiroglu S, van Karnebeek CD. Cerebral creatine deficiencies: A group of treatable intellectual developmental disorders. Semin Neurol 2014;34:350-6

30. Cervera-Acedo C, Lopez M, Aguirre-Lamban J, Santibañez P, GarciaOguiza A, Poch-Olive ML, et al. A novel SLC6A8 mutation associated with motor dysfunction in a child exhibiting creatine transporter deficiency. Hum Genome Var 2015;2:15037.

31. Rooms L, Reyniers E, van Luijk R, Scheers S, Wauters J, Ceulemans B, et al. Subtelomeric deletions detected in patients with idiopathic mental retardation using multiplex ligation-dependent probe amplification (MLPA). Hum Mutat 2004;23:17-21.

32. Sogaard M, Tümer Z, Hjalgrim H, Hahnemann J, Friis B, Ledaal P, et al. Subtelomeric study of 132 patients with mental retardation reveals 9 chromosomal anomalies and contributes to the delineation of submicroscopic deletions of 1pter, 2qter, 4pter, 5qter and 9qter. BMC Med Genet 2005;6:21

33. Erjavec-Skerget A, Stangler-Herodez S, Zagorac A, Zagradisnik B, Kokalj-Vokac N. Subtelomeric chromosome rearrangements in children with idiopathic mental retardation: Applicability of three molecularcytogenetic methods. Croat Med J 2006;47:841-50.

34. Wang S, Pan H, Pei P, Zheng X, Zhang Y, Ma Y, et al. Multiplex ligation-dependent probe amplification for detecting submicroscopic chromosomal abnormalities in Chinese children with global developmental delay or intellectual disability. Zhonghua Yi Xue Za Zhi 2014;94:2514-8 